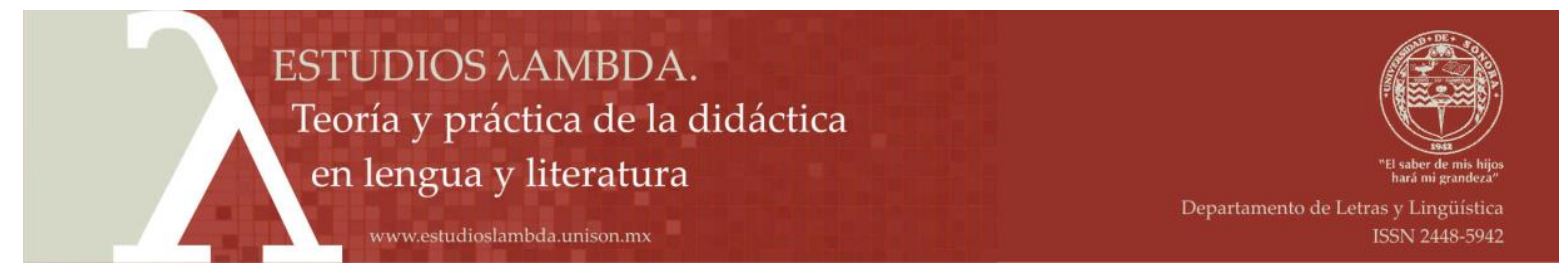

\title{
Alfabetización académica y disciplinar: intervención con estudiantes de Doctorado en Educación
}

(Academic and disciplinary literacy: intervention with doctorate students in Education)

\author{
Melanie Elizabeth Montes Silva ${ }^{\mathbb{D}}$, CETYS Universidad, Baja California, México \\ José Luis Bonilla Esquivel ${ }^{\mathbb{D}}$, CETYS Universidad, Baja California, México \\ Edna Mireya Salazar Robles ${ }^{(\mathbb{D},}$, CETYS Universidad, Baja California, México
}

\author{
Volumen 4, Número 1 \\ Enero-Junio de 2019 \\ p. $47-70$
}

Este número se publicó el 30 de junio de 2019

Artículo recibido: 6 de julio de 2018

Artículo aceptado: 30 de mayo de 2019

ISSN: 2448-5942 doi: https://doi.org/10.36799/el.v4i1.84

Citar este artículo como:

Montes Silva, M. E., Bonilla Esquivel, J. L., y Salazar Robles, E. M. (2019). Alfabetización académica y disciplinar:

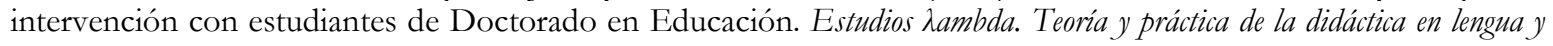
literatura. , 4(1), 47-70. https://doi.org/10.36799/el.v4i1.84

Derechos de autor: El autor o autores conservan en todo momento sus derechos morales y patrimoniales sobre la obra; la obra no se puede alterar, transformar o ampliar; siempre debe reconocerse la autoría del documento referido. Ninguna de las modalidades de los documentos publicados en Estudios $\lambda a m b d a$. Teoría y práctica de la didáctica en lengua y literatura tienen fines comerciales de naturaleza alguna.

Los contenidos de este artículo están bajo una licencia de Creative Commons Atribución No Comercial- Sin Derivadas 4.0 Internacional 


\title{
Alfabetización académica y disciplinar: intervención con estudiantes de Doctorado en Educación
}

\author{
Academic and disciplinary literacy: intervention with doctorate \\ students in Education
}

\author{
Melanie Elizabeth Montes Silva ${ }^{1}$ \\ JOSÉ LUIS BONILLA ESQUIVEL ${ }^{2}$ \\ EDNA MIREYA SALAZAR ROBLES ${ }^{3}$
}

\section{RESUMEN}

Los estudiantes de un Doctorado en Educación carecían de formación y experiencia para redactar textos académicos; además, sus anteproyectos iniciales mostraron deficiencias según el género. Se implementó una intervención, basada en la concepción de lectura y escritura de los Nuevos Estudios de Literacidad y en la noción de formar explícitamente para propiciar la participación en prácticas propias de cada ámbito. Los objetivos de la intervención fueron acompañar a los estudiantes, hacerlos conscientes de las características de la escritura académica y dotarles de herramientas. Tuvo dos etapas: un curso extracurricular sobre escritura académica y la asignación de una tutora de escritura para retroalimentar y evaluar los trabajos asignados en una materia curricular. Para documentar la experiencia se registró la opinión de los estudiantes, se entrevistó a los facilitadores de la intervención y a los titulares de las materias, y se aplicó un cuestionario a tres estudiantes. Los doctorandos consideraron que las actividades les ayudaron a tomar conciencia de leer de manera constante y crítica, y escribir según los lineamientos de la comunidad disciplinar en que se forman. Los profesores detectaron un cambio de actitud, el desarrollo de habilidades para la escritura y la autorregulación por parte de los estudiantes; los estudiantes proponen como mejora que la intervención sea más práctica y que se sitúe cuando ya están elaborando la tesis. Se valora positivamente la intervención y se identifica la necesidad de continuar con la práctica constante de la lectura y escritura para propiciar el desarrollo de la literacidad disciplinar.

PALABRAS CLAVE: alfabetización académica, literacidad disciplinar, cursos de escritura académica, estudiantes doctorales.

\begin{abstract}
Students from a Ph.D. in Education lacked training and experience to write academic texts; in addition, their preliminary projects showed deficiencies on the characteristics of the genre. An intervention was deployed, based on the New Literacy Studies and the notion that training must be explicitly implemented to promote participation in practices of each area. The objectives of the intervention were to accompany the students, make them aware of the characteristics of academic writing and to equip them with tools. It had two stages: an extracurricular course on academic writing and the assignment of a writing tutor, to provide feedback and to assess the tasks assigned in a curricular course. In order to document the experience, the student's opinion was registered, interviews were conducted with the instructors of the courses and the intervention, and a questionnaire was applied to three students. The doctoral students considered those activities helped them to become aware of the importance of constant and critical reading, and of writing according to their disciplinary community guidelines. Professors detected a change in attitude, the development of writing skills and self-regulation by the students. The students propose as an improvement that the intervention is more practical and that it is placed when
\end{abstract}

\footnotetext{
${ }^{1}$ Melanie Elizabeth Montes Silva es comunicóloga, así como doctora en ciencias educativas por el IIDE-UABC. Se desempeña como profesora de tiempo completo en CETYS Universidad, campus Tijuana, en Baja California, México. Correo electrónico: melanie.montes@,cetys.mx, ORCID: https://orcid.org/0000-0002-0499-0537

${ }^{2}$ José Luis Bonilla Esquivel es filósofo, así como doctor en educación por el ITESO. Se desempeña como profesor de tiempo completo en CETYS Universidad, campus Tijuana, en Baja California, México. Correo electrónico: joseluis.bonilla@,cetys.mx, ORCID: https://orcid.org/0000-0003-1944-857X

${ }^{3}$ Edna Mireya Salazar Robles es educóloga, así como maestra en administración de instituciones educativas por la UDLAP. Se desempeña como coordinadora del departamento de Formación Integral del Profesorado en CETYS Universidad, campus Tijuana, en Baja California, México. Correo electrónico: mireya.salazar@cetys.mx, ORCID: https://orcid.org/0000-0002-2133-1875
}

Artículo recibido: 6 de julio de 2018

Artículo aceptado: 30 de mayo de 2019

Montes, Bonilla y Salaz̧ar doi: https://doi.org/10.36799/el.v4i1.84 Volumen 4, Número 1, Año 2019, ISSN: $2448-5942$ 
they are already preparing the thesis. The intervention is positively valued despite starting as a remedial activity, and the need to continue with the constant practice of reading and writing to promote the development of disciplinary literacy in the students is identified.

KEY WORDS: academic literacy, disciplinary literacy, academic writing courses, doctoral students.

\section{INTRODUCCIÓN}

Es posible sostener que lo que se aprende sobre lectura y escritura en la infancia no es suficiente para atender todos los retos que impondrá la vida académica; hay distintos modos de leer y de escribir, y no es posible transferir de manera automática todos los conocimientos y habilidades que se tienen para resolver exitosamente distintos tipos de tareas (Carlino, 2003). Tomando esto en consideración es comprensible que en el ámbito de la educación formal sea necesario ofrecer formación explícita sobre lo que se espera que los estudiantes hagan en cada nivel y en cada contexto. Esto, sobre todo, porque conforme los estudiantes avanzan en su vida académica deben comprender y producir textos más abstractos y especializados, que tienen cada vez menos paralelismos con su vida cotidiana y que requieren de rutinas más sofisticadas; además, se enfrentan al hecho de que las habilidades y los conocimientos que requieren suelen no ser enseñados, pues muchos profesores asumen que al llegar a niveles avanzados ya saben leer y escribir cualquier tipo de texto que se les exija (Shanahan y Shanahan, 2008) y que su única tarea es aplicar sus conocimientos a otros contextos y a otros contenidos (Camps, 2007).

La lectura y la escritura no son solo tareas escolares, sino que tienen el potencial para lograr que los estudiantes construyan conocimientos, razonen, reflexionen, transformen y autorregulen su aprendizaje, no solo sobre la lectura y la escritura en sí mismas, sino sobre lo que leen y escriben; es decir, la lectura y la escritura tienen una función epistémica. (Applebee, 1984; Carlino, 2006; Miras, 2014; Olson, 1996; Serrano, 2014). Además, la lectura y la escritura deben concebirse relacionadas y como parte del proceso formativo, no solo como el resultado final.

Leer, escribir, aprender y comunicar son actividades interdependientes y estrechamente relacionadas. Desde esta perspectiva, la composición de textos académicos, la escritura científica, no puede entenderse como el mero producto del proceso de aprendizaje e indagación, como el resultado o el punto final del mismo, sino como uno de los elementos integrantes del proceso mismo, a lo largo del cual los usos de la lectura y la escritura van variando y cumplen funciones diversas (Miras y Solé, 2007, 85). 
Los estudios de doctorado no son una excepción a esta situación. A los doctorandos se les exige la producción de textos académicos especializados en un campo disciplinario, los cuales deben tener sustento en una investigación original (Carrasco Altamirano y Kent Serna, 2011). Además, estos textos deben apegarse a ciertos lineamientos preestablecidos, pues "las comunidades académicas han convencionalizado los modos de comunicación disciplinar a través de los géneros científico-académicos y de los modos de organización típicos" (Padilla, 2016, 169). En algunos programas se solicita que los estudiantes realicen ponencias o artículos derivados de sus investigaciones, además de monografías u otro tipo de textos elaborados como parte de su proceso formativo; no obstante, el texto más destacado en el doctorado es la tesis, cuya elaboración no es una tarea sencilla, pues algo que le caracteriza es que "exige una gran autoorganización y que carece de parangón con cualquier otra tarea académica antes intentada, al menos para quienes la inician sin experiencia en investigación” (Carlino, 2005, 2).

Por esto, también en el doctorado se hace necesario ofrecer formación explícita sobre cómo escribir una tesis, en especial porque las orientaciones que ofrecen las instituciones suelen limitarse al establecimiento de pautas sobre aspectos más bien generales, que no especifican qué se espera del trabajo de los estudiantes (Carlino, 2008). Así, la tarea formativa suele recaer principalmente en el tutor, quien acompaña directamente al estudiante en el proceso de la investigación, redacción y presentación de la tesis; no obstante, los modos de asesorar a los doctorandos varían significativamente. Puede ocurrir que los directores se centren en la tarea, que se interesen por enculturar a los estudiantes en una comunidad de práctica o que los acompañen en ámbitos personales; puede ocurrir cualquier forma de dirección entre dos extremos: que el director tome todas las decisiones metodológicas o que el director se muestre indiferente (Fernández Fastuca y Wainerman, 2015). Este proceso puede llegar a generar tensiones entre los directores y los doctorandos, especialmente si se consideran las presiones institucionales a las que ambos están expuestos y las metas que cada uno tiene (Carter y Kumar, 2016). Visto así, es un reto lograr cumplir con el compromiso de las instituciones de formar a los estudiantes de doctorado para que lean y escriban conforme a lo esperado en la comunidad de práctica en la que están siendo formados, asumiendo que lo que han hecho antes no necesariamente los dota de los conocimientos y las habilidades que requieren para elaborar una tesis de grado, con todo lo que ello implica. 
En este trabajo se presenta una intervención llevada a cabo con estudiantes de un programa de Doctorado en Educación. La finalidad fue dotar a los doctorandos de herramientas que les dieran elementos para afrontar los primeros cursos curriculares del programa, así como elaborar sus anteproyectos de investigación. Los contenidos se centraron tanto en aspectos básicos y generalizables, como en otros especializados para su nivel educativo y ámbito de formación.

\section{MARCO DE REFERENCIA}

Desde la perspectiva de los Nuevos Estudios de Literacidad (NEL) (Gee, 2000; Street, 2013) debe entenderse la literacidad como una actividad social que involucra la forma en la que las personas usan la lectura y la escritura, tanto para participar en prácticas culturales, como para cumplir propósitos sociales más amplios (Zavala, 2008). Estas prácticas pueden tener lugar en diferentes contextos, ya sean informales, formales o institucionalizados, y tienen características diferentes según la situación. En el ámbito de la educación formal se puede hablar de la literacidad académica, que implica usar conocimientos y habilidades generales para resolver distintas tareas (como estrategias genéricas de comprensión), y la literacidad disciplinar, que son conocimientos y habilidades particulares para leer y escribir en cada una de las disciplinas (como Historia, Química o Matemáticas) (Shanahan y Shanahan, 2008). Algo importante en cuanto a la literacidad disciplinar es que la participación en prácticas de lectura y escritura conlleva aspectos diversos:

Remite básicamente a la participación activa de los individuos en la cultura de lo escrito, es decir, el conocimiento y reconocimiento del código escrito, de las reglas lingüísticas que gobiernan la escritura y de las convenciones establecidas para el texto. También se refiere al conocimiento y reconocimiento de géneros discursivos, de los roles que desempeñan los interlocutores en la comunicación escrita, de las formas de pensamiento y procedimientos de observación de la realidad, de la presentación de información y de razonamiento asociados con el discurso escrito; a la identidad y el estatus que los miembros de una comunidad han adquirido a través del uso de la escritura, y a los valores y marcos culturales que se elaboran a través del discurso escrito (Castro Azuara y Sánchez Camargo, 2015, 53). 
Al término de literacidad se asocia el de alfabetización, que hace referencia al proceso pedagógico que busca promover que los estudiantes participen en las prácticas que les demanda la institución educativa (alfabetización académica) o en las propias de la disciplina en la que están siendo formados (alfabetización disciplinar). Este tipo de formación tiene dos objetivos: “enseñar a participar en los géneros propios de un campo del saber y enseñar las prácticas de estudio adecuadas para aprender en él" (Carlino, 2013, 370); es decir, aprender no solo a leer y a escribir como lo hacen los especialistas de cada área, sino también, aprender los conocimientos propios de cada disciplina. Así, podrá ser posible que los estudiantes comprendan el conocimiento que se ha acumulado con el tiempo en una disciplina concreta, sepan cómo se produce ese conocimiento y desarrollen la capacidad de producirlo y criticarlo (Moje, 2007).

Las instituciones educativas implementan diferentes estrategias para promover la alfabetización académica. Calderón Arévalo y Tapia Ladino (2016) indagaron sobre cuáles son las actividades académicas para el desarrollo de la lectura y la escritura que llevan a cabo universidades de Chile, México, Colombia, Argentina, Brasil, Venezuela y Puerto Rico e identificaron ocho iniciativas. Algunas son resultado de esfuerzos docentes individuales, mientras que otras cuentan con apoyo institucional, y por consecuencia, tienen mayor alcance, cobertura y legitimación. Enseguida se presentan estas iniciativas, organizadas desde las que son más institucionalizadas a las que tienen menos respaldo institucional, según la clasificación de las autoras referidas.

1. Cursos intensivos sobre lectura, escritura u oralidad. Se refiere a los cursos curriculares centrados en estos contenidos que se ofrecen en las universidades.

2. Centros de apoyo pedagógico orientados al aprendizaje de lectura, escritura y oralidad. En estos centros hay personal especializado que asesora a estudiantes y a profesores que lo requieren, también diseñan material didáctico.

3. Centro de estrategias de evaluación. Integrado por especialistas que se encargan de evaluar y dar seguimiento al aprendizaje y a las habilidades de los estudiantes sobre lectura, escritura u oralidad.

4. Programas de ayudantías o compañeros de lectura, escritura u oralidad. Estudiantes que han demostrado tener conocimientos y habilidades, generalmente de cursos superiores, que son seleccionados para orientar a sus compañeros como parte de las actividades de una materia en concreto e interactúan en contextos informales. 
5. Sistema de tutores. Son también estudiantes avanzados que orientan a otros estudiantes que lo requieren, pero la interacción ocurre en contextos académicos más formales y no se da en el marco de una materia en concreto.

6. Recursos pedagógicos en línea. Estos recursos son creados por personal de la institución, están disponibles en internet y son guías para estudiantes y maestros.

7. Pareja pedagógica. La pareja está conformada por dos académicos que trabajan colaborativamente con un mismo grupo; uno es experto en una disciplina y se dedica a trabajar los contenidos propios de la materia, el segundo es especialista en el desarrollo de habilidades del lenguaje y da seguimiento constante al progreso de las habilidades de los estudiantes en cuanto a escritura, comprensión de la lectura, comunicación oral y el dominio de las convenciones propias del área de conocimiento.

8. Seminarios transversales o emergentes. Son cursos o talleres que se dictan al inicio o al final de un periodo para abordar cuestiones concretas que requieran los estudiantes.

Como se puede ver, las iniciativas son de distintos tipos y, aunque cumplen propósitos similares, los acercamientos son diferentes. Además, este tipo de iniciativas han tenido reacciones diversas. Por ejemplo, los seminarios transversales que se dan en el inicio de un periodo lectivo, generalmente de forma remedial, suelen ser criticados, pues la enseñanza no se da siempre de manera situada y como herramienta epistémica, como puede esperarse en el marco de los NEL, sino que se ven de manera tradicional, centrados en la corrección idiomática con fines de nivelación (Ávila Reyes, González-Álvarez y Peñaloza Castillo, 2013).

Lo relevante de estas iniciativas es que son un marco de referencia para situar la intervención realizada con relación a la alfabetización académica y disciplinar entre estudiantes de doctorado, cuyo contexto e implementación se describe enseguida.

\section{ESCENARIO Y PARTICIPANTES}

El contexto en el que tiene lugar la intervención es el de una institución educativa privada, específicamente en el marco de la primera generación del programa de Doctorado en Educación. La convocatoria para ingresar al programa establecía que para ser admitidos los aspirantes debían presentar un examen de admisión y un anteproyecto de investigación, además de sostener una Montes, Bonilla y Salazar doi: https://doi.org/10.36799/el.v4i1.84 Volumen 4, Número 1, Año 2019, ISSN: $2448-5942$ 
entrevista con personal docente de la institución. Todos los estudiantes que cumplieron con los requisitos fueron admitidos, sin tomar en cuenta su formación previa, su experiencia en investigación ni la dedicación que tendrían al programa, pues no es de dedicación exclusiva.

En el caso del examen de admisión, se utilizó la Prueba de Admisión a Estudios de Posgrado (PAEP) (Instituto Tecnológico y de Estudios Superiores de Monterrey, 2014), la cual considera la evaluación del razonamiento verbal y de la redacción, entre otros aspectos. En la tabla 1 se puede ver una síntesis de los resultados obtenidos en la prueba por los doctorandos admitidos. Ahí destaca que hay puntuaciones bajas, sobre todo considerando que la misma institución establece que para que un estudiante sea admitido a un programa de maestría debe tener mínimo 400 puntos; en caso de que obtenga entre 350 y 399 puede ser admitido de manera condicional y, si obtiene 349 puntos o menos, el aspirante es rechazado. En el caso del Doctorado en Educación, la media del puntaje final de los aceptados fue de 458.06 y cuatro estudiantes quedaron por debajo de los 400 puntos. Dada la naturaleza predictiva de la prueba, estos resultados representan un foco de alarma a tener en consideración. 
Tabla 1. Resultados obtenidos por los estudiantes del doctorado en el examen de admisión

\begin{tabular}{lcccc}
\hline \multicolumn{1}{c}{ Aspecto a evaluar } & Mínimo & Máximo & Media & $\begin{array}{c}\text { Desviación } \\
\text { estándar }\end{array}$ \\
\hline Razonamiento verbal & 37 & 66 & 48.41 & 8.52 \\
Razonamiento matemático & 31 & 63 & 43.24 & 8.73 \\
Habilidad cognoscitiva & 29 & 69 & 48.53 & 10.85 \\
Redacción & 32 & 66 & 51.29 & 15.56 \\
Inglés & 32 & 97 & 47.41 & 9.85 \\
Puntaje final & 339 & 561 & 458.06 & 67.62 \\
\hline
\end{tabular}

${ }^{1} \mathrm{La}$ escala de las puntuaciones de cada sección es de 20 a 80, mientras que la escala de la puntuación final es de 200 a 800; además, el puntaje final se determina con una ponderación de los puntajes burdos obtenidos en cada sección de la prueba (Instituto Tecnológico y de Estudios Superiores de Monterrey, 2004).

Fuente: elaboración propia.

En cuanto a los anteproyectos solicitados, en la convocatoria no se especificaron criterios que los candidatos debieran atender, lo cual provocó que los documentos que presentaron los aspirantes fueran disímiles. La extensión varió entre dos y hasta 45 cuartillas; había documentos cuyo alcance se limitaba a plantear un problema y otros que presentaban instrumentos desarrollados o incluso datos exploratorios para sustentar la propuesta; había trabajos con fundamentación teórica y otros que carecían de sustento en fuentes de información; los elementos del diseño metodológico que se incluían eran diferentes y su consistencia no siempre era clara; los formatos empleados eran diferentes o no se seguía alguno en particular; en general, no había consistencia en cuanto a las características del género textual protocolo de investigación.

En la tabla 2 se sintetizan las características de los 17 estudiantes admitidos al programa de doctorado. Como se puede ver, el grupo resultó ser heterogéneo en cuanto a su formación de licenciatura, aunque había más uniformidad en los estudios de maestría: la mayoría tiene el grado de maestría en Educación, todos cursaron un programa profesionalizante y ninguno hizo una tesis como opción de titulación. También coincidían en que todos tenían experiencia en docencia, aunque en diferentes niveles educativos; algunos también habían trabajado en ámbitos vinculados con la gestión educativa. Solo dos personas señalaron tener experiencia en la realización de investigación educativa y en la difusión del conocimiento generado, el resto tenía nociones de metodología de la investigación solo por las clases que había tomado.

Tabal 2. Perfiles de los estudiantes admitidos al Doctorado en Educación 


\begin{tabular}{|c|c|c|c|}
\hline Edad & $\begin{array}{l}\text { Área de formación: } \\
\text { licenciatura }\end{array}$ & $\begin{array}{c}\text { Área de formación: } \\
\text { maestría }\end{array}$ & $\begin{array}{l}\text { Experiencia principal en } \\
\text { el campo educativo }\end{array}$ \\
\hline $\begin{array}{l}\text { Mínima: } 27 \\
\text { Máxima: } 54 \\
\text { Media: } 39\end{array}$ & $\begin{array}{l}\text { Educación: } 5 \\
\text { Comunicación: } 3 \\
\text { Psicología: } 4 \\
\text { Ingeniería: } 2 \\
\text { Administración: } 1 \\
\text { Derecho: } 1 \\
\text { Medicina: } 1\end{array}$ & $\begin{array}{l}\text { Educación: } 14 \\
\text { Administración: } 2 \\
\text { Ciencias jurídicas: } 1\end{array}$ & Docente: 17 \\
\hline
\end{tabular}

Fuente: elaboración propia.

Una vez que concluyó el proceso de admisión y cuando se acercaba el inicio de clases, la institución optó por cambiar al coordinador académico. El nuevo responsable del programa analizó las características de los estudiantes que ya habían sido admitidos y escuchó las opiniones de la doctora que estaba impartiendo el primer curso curricular, principalmente en el sentido de que los estudiantes manifestaban no estar dispuestos a leer y no tenían habilidades en cuanto a la escritura de textos académicos. Con base en estos elementos el coordinador del programa decidió implementar una intervención para apoyar a los estudiantes que requerían desarrollar habilidades para cursar con éxito el programa. Por cuestiones de tiempo y por las circunstancias en las que se detectó la necesidad, las actividades debían ser remediales y comenzaron, en su primera etapa, cuando los estudiantes ya habían iniciado las actividades propias del programa. El plan de estudios del programa está estructurado para que en el primer semestre se cursen dos materias teóricas sobre nociones educativas; en el segundo, dos materias sobre métodos de investigación cualitativos y cuantitativos, y en el tercer semestre se comience con el proyecto de tesis. Por lo anterior, la intervención debía dotar a los estudiantes de las herramientas básicas para que pudieran realizar las actividades propias de las asignaturas y, además, el protocolo de investigación de su tesis.

La intervención contó con la participación de dos facilitadoras. Quien trabajó la primera etapa tiene el grado de doctor y su formación es en Educación y en Lingüística; quien trabajó la segunda etapa tiene el grado de maestría y su formación es en Educación. Ambas instructoras trabajaron previamente en un centro de escritura y tienen experiencia en el acompañamiento de estudiantes sobre cuestiones de redacción académica. Para la recolección de información se documentó la experiencia de cada facilitadora y se realizaron entrevistas con ellas; adicionalmente, se entrevistó a dos de los docentes titulares de cursos curriculares impartidos y Montes, Bonilla y Salazar doi: https://doi.org/10.36799/el.v4i1.84 Volumen 4, Número 1, Año 2019, ISSN: $2448-5942$ 
se solicitó la opinión de tres estudiantes, a través de un cuestionario de preguntas abiertas, a fin de conocer su valoración sobre la experiencia. Cabe precisar que los tres estudiantes fueron seleccionados de manera intencional, pues según información proporcionada por las facilitadoras, estos estudiantes podían ofrecer perspectivas diferentes de los resultados.

\section{CARACTERÍSTICAS GENERALES DE LA INTERVENCIÓN}

La intervención tuvo dos etapas: (1) un curso extracurricular sobre escritura académica, que tuvo una duración de 21 horas presenciales, distribuidas en siete sesiones semanales, además de 14 horas de trabajo independiente, y (2) la asignación de una tutora de escritura que trabajó como pareja pedagógica del profesor de una materia curricular, específicamente para orientar a los estudiantes, así como retroalimentar y evaluar los trabajos asignados como parte de la asignatura.

\subsection{PRIMERA ETAPA DE LA INTERVENCIÓN: CURSO EXTRACURRICULAR}

El curso extracurricular se llamó Desarrollo de literacidad académica y disciplinar, y se impartió mientras los estudiantes aún cursaban la primera materia curricular del programa y cuando todavía no comenzaban a trabajar en su anteproyecto de tesis. Por ser una actividad extraordinaria, la participación de los doctorandos fue voluntaria. A ellos se les presentó como un taller sobre cuestiones de escritura académica y, aunque hubo estudiantes a los que les pareció una actividad oportuna, hubo otros que cuestionaron la pertinencia de un curso de este tipo, pues les parecía que no lo necesitaban por el nivel de estudios que estaban comenzando; también hubo quienes comenzaron a dudar de sus habilidades, pues asumieron que si se ofrecía el curso era porque alguien había considerado que lo requerían. A pesar de sus dudas iniciales, la mayoría asistió; se contó con la presencia constante de 13 de los 17 estudiantes inscritos al programa.

La actividad comenzó con un encuadre, en el que se presentaron los participantes, se indagaron sus expectativas y se describió la dinámica general que se seguiría. Posteriormente, se repartieron fragmentos de textos en los que se definían la alfabetización académica y la literacidad (Carlino, 2013; Fang, 2012); se señalaba que son prácticas sociales (Zavala, 2008); se describían los distintos tipos que hay y se justificaba la importancia de ofrecer formación explícita en torno a la lectura y la escritura (Shanahan y Shanahan, 2008); se describían los elementos que incluyen Montes, Bonilla y Salazar doi: https://doi.org/10.36799/el.v4i1.84 Volumen 4, Número 1, Año 2019, ISSN: $2448-5942$ 
estas nociones y se precisaba que las prácticas de literacidad difieren según el contexto y la disciplina en la que tengan lugar (Castro Azuara y Sánchez Camargo, 2015). Para guiar la lectura se ofrecieron preguntas orientadoras y, cuando terminaron de leer, se respondieron esas mismas interrogantes, con base en las aportaciones de todos. El ejercicio sirvió para problematizar las nociones de lectura y escritura, así como para que ellos pudieran justificar, con base en la teoría, la pertinencia del curso, lo que fue benéfico para su autoestima. El ejercicio también posibilitó clarificar que el curso buscaba contribuir al desarrollo de diversas literacidades (académica, disciplinar, informacional, visual, digital o ciberliteraciad y fluidez informativa) y habilidades (metacognición, autorregulación, transferencia, pensamiento crítico, competencia comunicativa y conciencia comunicativa), con miras a que tuvieran más y mejores elementos, en un principio, para elaborar el anteproyecto de su trabajo de tesis. Adicionalmente, se presentaron las finalidades del curso, las cuales fueron las siguientes:

- Contribuir al desarrollo de literacidades para que los estudiantes puedan participar en las prácticas propias del programa de Doctorado en Educación.

- Desarrollar conciencia comunicativa en los participantes como cimiento para que trabajen en el desarrollo de su competencia comunicativa.

El siguiente momento del encuadre consistió en presentar los contenidos del curso, pero para definirlos se realizaron una serie de actividades. Primero se proyectó una figura muy sencilla en la que se señalaban cuatro componentes del texto: ortografía, redacción, formato y fuentes de información. Se solicitó que identificaran qué aspectos concretos se desprendía de cada componente; los participantes anotaron uno o dos elementos de cada uno. Enseguida se les invitó a profundizar sobre el tema y para ello se ilustró lo relacionado con la ortografía. Se proyectó un párrafo muy breve y se les pidió que identificaran todos los errores de ortografía que pudieran localizar; en general les pareció un ejercicio muy sencillo y lo resolvieron con rapidez y seguridad. La cantidad de errores identificados varió, pero se sorprendieron al saber que un párrafo tan breve tenía 15 errores de distintos tipos y que nadie los había encontrado todos. Esto sirvió para invitarlos a pensar de una manera más compleja y a problematizar el tema con más seriedad, también dio pauta para hacer una lista más extensa de los componentes de cada elemento señalado con anterioridad.

El paso siguiente fue hacer comentarios sobre los anteproyectos de investigación que previamente se les había solicitado leer a manera de tarea; estos trabajos eran auténticos y fueron Montes, Bonilla y Salazar doi: https://doi.org/10.36799/el.v4i1.84 Volumen 4, Número 1, Año 2019, ISSN: $2448-5942$ 
elaborados por otros estudiantes de doctorado, pero como eran las versiones iniciales, tenían errores significativos. Se les pidió identificar los aciertos y las fallas de esos anteproyectos, con base en los aspectos discutidos en clase. Posteriormente, se les pidió señalar qué querían aprender en el curso, tomando en cuenta lo que veían en los otros trabajos y en lo que sabían de sí mismos. Pasaron al pizarrón y anotaron los siguientes temas: (1) ortografía; (2) redacción; (3) producir ideas estructuradas y que tengan fluidez; (4) esquemas de redacción académica; (5) superar el miedo a la redacción; (6) formato de la American Psychological Association (APA); (7) mejorar habilidades para buscar información, y (8) aumentar el vocabulario del doctorado. La facilitadora hizo preguntas sobre los temas anotados, para profundizar en sus expectativas, e hizo propuestas que los estudiantes aceptaron. De este modo se definió el temario del curso y su organización, de tal manera que los participantes se sintieron tomados en cuenta y consideraron que los temas se adecuaban a sus necesidades.

Las siguientes sesiones se desarrollaron con base en el temario establecido colectivamente y siempre se trabajó con un componente teórico y otro práctico. Algunos ejercicios fueron elaborados a partir de textos diseñados expresamente para ejemplificar algún tema, para otros se solicitó a los estudiantes que tuvieran consigo los anteproyectos que presentaron como requisito para ingresar al doctorado, de tal forma que los pudieran analizar a partir de los temas revisados y reescribieran o corrigieran algunas secciones. También se asignaron actividades extraclase, ya fuera para profundizar en los temas abordados o para preparar recursos que iban a ser necesarios para el desarrollo de alguna sesión.

Durante la segunda sesión se abordó el tema de la ortografía, con especial énfasis en la puntuación. Durante la tercera sesión se trabajó el tema de alfabetización informacional, para lo que se revisaron las bases de datos con las que cuenta la institución y se les enseñó a buscar, evaluar y almacenar la información relacionada con sus temas de interés. En la cuarta sesión se abordaron varios temas vinculados con la redacción: géneros y tipos de textos; componentes del anteproyecto; planeación del texto en general y de los apartados del anteproyecto en particular. La quinta sesión también fue sobre redacción, pero centrada en otros aspectos: oraciones, párrafos y textos; coherencia y cohesión; marcadores discursivos. La sexta sesión abordó lo relacionado con el manual de publicaciones de la APA. En la séptima y última sesión se trabajó el tema de los vicios del lenguaje y se practicaron dos recursos digitales: el gestor de bibliografía Zotero y el procesador de texto Word, específicamente para revisar funciones avanzadas. 
Durante el desarrollo del curso los estudiantes expresaron sorpresa ante los temas abordados. Comentaron no saber que existían tantos aspectos sobre los que debían prestar atención y señalaron darse cuenta de lo que les hacía falta aprender. Era común que hablaran sobre las fallas que habían detectado en sus anteproyectos iniciales (de aspectos normativos, estilísticos y metodológicos), incluso a pesar de que en su momento los habían considerado como muy buenos. También llegaron a cuestionarse acerca de la razón por la que los habían aceptado en el programa, en especial si tenían tantas carencias, aunque lo señalaban en tono de broma. El ánimo siempre fue bueno y la disposición era adecuada para el trabajo.

En cuanto al resultado del curso, la facilitadora identificó, a partir de las intervenciones de los estudiantes durante el desarrollo de las actividades, que comenzaron a desarrollar conciencia comunicativa, en el sentido de que tenían más sensibilidad para identificar aspectos por mejorar con relación a lo normativo, aunque no tenían las competencias necesarias para hacer todas las correcciones que el texto requería. Asimismo, a partir de la valoración que los participantes hicieron durante el cierre del curso, se evidenció una mayor apertura al hecho de que las formas de leer y de escribir debían ser diferentes en el contexto formativo en el que se encontraban. Identificaron que las formas de leer y de escribir de sus áreas de formación previa eran peculiares y que, por tanto, no podían transferirlo a lo que ahora se les solicitaba en el doctorado; lo mismo ocurría con sus prácticas vernáculas y con las que solían realizar en el contexto de su práctica profesional, por ejemplo, algunos señalaron el hecho de que en los textos que solían escribir para su trabajo no era necesario referir las fuentes consultadas o seguir un manual de estilo en particular, incluso quienes trabajaban en ambientes académicos. Tomaron conciencia de las razones por las que debían leer como un medio para aprender sobre los temas que iban a investigar y sobre cómo investigarlos, aunque en principio estaban renuentes al hecho de leer; también comprendieron que la escritura es fundamental en su formación doctoral y que debían escribir para un lector experto y no para sí mismos, aunque al inicio también manifestaron poco interés con respecto a la escritura. Finalmente, durante el cierre del taller, los doctorandos señalaron haber comprendido que estaban comenzando un proceso de enculturación en un área particular y que el rol de los tutores sería crucial, por lo que debían apegarse a sus lineamientos y seguir sus recomendaciones.

\subsection{SEGUNDA ETAPA DE LA INTERVENCIÓN: PAREJA PEDAGÓGICA}


Una vez desarrollado el curso extracurricular sobre escritura académica, como primera etapa de la intervención, se llevó a cabo un acompañamiento a los doctorandos por parte de una tutora de escritura, en conjunto con el profesor de la segunda asignatura del doctorado, titulada Escuela y sociedad en el mundo contemporáneo. Este curso buscó que se establecieran juicios sobre el papel de la educación en un ambiente de crisis social, cultural y paradigmático. La pareja pedagógica organizó en conjunto el trabajo del curso, las actividades a realizar y las funciones de cada uno: la tutora de escritura revisaría y retroalimentaría puntualmente los trabajos de los estudiantes en cuanto a aspectos normativos y estilísticos, además de que desarrollaría actividades para guiarlos; el profesor titular, por su parte, se encargaría de los contenidos propios de la asignatura, no solo durante la sesión, sino al retroalimentar los trabajos en cuanto a aspectos de contenido y argumentación académica.

El primer día de clases los tres estudiantes que no asistieron al curso extracurricular cuestionaron las implicaciones de este para su segunda materia, ya que por los comentarios de sus compañeros comprendieron que lo debían haber tomado, a pesar de que no era obligatorio. Ante esta situación se les solicitó que revisaran el material del curso extracurricular, disponible para todos los doctorandos en una plataforma educativa, y se acordó que la tutora podría orientarlos en caso de que tuvieran dudas sobre ello o sobre cualquier aspecto adicional. Además, la tutora les facilitó material complementario y, tanto a ellos como al resto de los estudiantes, les respondió dudas, sobre todo por correo electrónico.

La clase se desarrolló en seis sesiones, la primera consistió en diferentes momentos. Como inicio, el docente realizó el encuadre, donde detalló el programa de la materia y se prestó principal atención a la evidencia de desempeño que se debía entregar al concluir la asignatura. Esta consistió en la entrega de un texto cuyo género era de elección para los estudiantes, pues podían optar entre un ensayo académico, un relato o una narración que se fundamentara en la dimensión teórico/educativa de la ética de la responsabilidad. La elaboración del texto ayudaría a ir perfilando el interés investigativo de los estudiantes a partir de la realización de un documento escrito como evidencia del desarrollo de sus habilidades para la escritura y de la capacidad discursiva necesaria para la elaboración de su tesis. Posteriormente, la tutora de escritura platicó con los doctorandos sobre su función dentro de la clase, así como las cuestiones generales de formato y presentación mínimamente esperadas en sus trabajos escritos, con base en los aspectos revisados en el curso extracurricular que la mayoría había tomado, acompañado de aquellas 
habilidades que un programa de doctorado requiere. Dichas cuestiones se basaron en que todos los documentos que se les solicitarían como parte del curso debían incluir los siguientes elementos básicos de presentación:

- Redacción clara, coherente, cohesiva, donde se evidenciara concordancia, adecuación, variedad y precisión léxica.

- Uso correcto de la ortografía.

- Signos de puntuación correctamente empleados.

- Congruencia intra e inter-párrafo, presentando una idea principal y alrededor de tres ideas secundarias, y que se guardara concordancia entre las ideas y los párrafos.

- Estructura lógica y ordenada sobre las secciones y los encabezados.

- Citas, paráfrasis, referencias y aspectos generales de presentación de acuerdo al manual de la APA.

Una vez establecidos los lineamientos con los que debían contar sus trabajos, se estableció la calendarización de evidencias de desempeño a entregar. Un aspecto que llamó la atención de la primera sesión fue que los doctorandos manifestaron una expectativa con respecto al curso: Ojalá que no leamos mucho, lo que se asemeja al comentario de la profesora que impartió la primera materia.

Para la segunda sesión se pidió la entrega de una reflexión sobre el caso de Frank McCourt, la cual permitió conocer las habilidades de escritura con las que contaban los doctorandos. Como resultado se identificó la carencia de habilidades para escribir particularmente en uno de los estudiantes, quien no había asistido al curso previo; el resto de los estudiantes mostraron un mejor ejercicio en cuanto a aspectos normativos y estilísticos, pero aún tenían aspectos por mejorar y la tutora lo señaló en la retroalimentación de los trabajos. Para la tercera sesión elaboraron un texto donde se identificaban cuatro ideas o planteamientos teóricos del texto Una escuela con rostro bumano (Ortega Ruiz y Gárate Rivera, 2017), lo que permitió a los estudiantes mejorar en aquellos aspectos que la tutora señaló en la retroalimentación de su primera evidencia y tener mayor práctica en la elaboración de trabajos escritos. Para la cuarta sesión se pidió que llevaran el primer avance de su trabajo final, que debían entregar en la sexta y última sesión. Con la entrega y revisión de borradores tenían elementos para mejorar su documento. 
Según lo que refiere la tutora de escritura, en los primeros trabajos entregados por los estudiantes se encontraron como áreas de mejora cuestiones de redacción, ortografía y paráfrasis. A pesar de que en muchas ocasiones hacían hincapié en que lo habían revisado en el curso de literacidad, decían que les hacía falta practicarlo, ya que en ocasiones pensaban que lo sabían, pero no estaban seguros de hacerlo bien. Por esto, un factor que se trabajó con ellos fue que confiaran en sus capacidades y habilidades, ya que eso en ocasiones les afectaba en el desarrollo de sus documentos. Para lograrlo, una de las estrategias utilizadas fue que en algunas sesiones leyeron los trabajos de sus compañeros. La retroalimentación de un par los motivó, además, posibilitó que ellos mismos detectaran sus áreas de oportunidad sin necesidad de que alguien más se las dijera. De esta manera se identificó un avance en la entrega de los trabajos finales, ya que fueron documentos mejor cuidados y limpios, con aspectos mínimos por mejorar en lo normativo y estilístico, según identificó la tutora de escritura al hacer la retroalimentación de los trabajos.

A lo largo de la asignatura era recurrente escuchar el nombre de la maestra que había facilitado el curso de literacidad. Recordaban los aprendizajes, comentarios y recomendaciones que les había dado la facilitadora para la elaboración de sus trabajos, lo que servía como un espacio para compartir ideas y reflexiones sobre algunas técnicas que les habían funcionado o algún material que seguían revisando, como era el manual de la APA, lo que servía tanto para aquellos que habían tomado el curso como para los que no. Esto, desde la perspectiva de la tutora de escritura, evidenció un aprendizaje significativo en los doctorandos.

Al ser la tarea de la tutora, en conjunto con el docente de la asignatura, orientar, retroalimentar y evaluar los trabajos escritos elaborados por los estudiantes, se pudo determinar que el nivel con el que se recibió en la primera sesión al grupo fue mejor de lo esperado. Además, la indicación de que debían presentar debidamente la información que consultaran, aunado a que se les proporcionaran retroalimentaciones puntuales sobre los trabajos, fueron acciones recibidas de manera positiva por parte de los doctorandos, pues los aspectos señalados no les eran ajenos, debido a que tenían conocimiento previo de ello. Generalmente se evidenciaban fallas por cuestiones de organización de tiempo, presión personal, falta de atención y práctica, por lo que se considera que el curso previo sí apoyó el desarrollo de habilidades y conocimientos por parte de los estudiantes. Lo anterior se respalda con las opiniones expresadas por los estudiantes en la evaluación que realizaron sobre el curso, donde señalan que, a través de la orientación efectiva Montes, Bonilla y Salazar doi: https://doi.org/10.36799/el.v4i1.84 Volumen 4, Número 1, Año 2019, ISSN: $2448-5942$ 
que recibieron, los trabajos realizados en la asignatura reforzaron la escritura de textos académicos, la estructura de ideas y la búsqueda de fuentes confiables de información. Además, conforme avanzó el curso, los doctorandos manifestaron más disposición para leer los textos asignados e incluso comenzaron a consultar fuentes adicionales, que servían para la discusión y para la elaboración de sus trabajos, y tuvieron menos miedo de escribir y de usar su voz en los trabajos.

Desde la perspectiva de la tutora de escritura, lo que hace falta es que los estudiantes tengan más práctica y que esta esté acompañada, porque después de la intervención los estudiantes están más empoderados, se sienten más seguros y confiados, pero saben qué les hace falta aprender y eso ayuda a que autorregulen más su proceso de aprendizaje. Según el doctor titular del curso curricular, hay dos aspectos sobre los que se debe seguir trabajando. El primero es el uso de la voz de los estudiantes en sus textos y la integración de otras voces mediante citas y paráfrasis. Con base en la revisión del contenido de los trabajos, él identificó que hay estudiantes que sí logran incorporar bien las citas al texto; otros las colocan de manera forzada, aparentemente por cumplir con un requisito, y hay todavía casos de estudiantes que fundamentan sus trabajos académicos únicamente con su experiencia personal. El segundo aspecto es que los estudiantes tienen que transitar un camino para volverse especialistas de un campo de conocimientos específicos, pues de momento hay estudiantes que no manejan el discurso educativo. En definitiva, a su juicio hay doctorandos que tienen elementos para seguir adelante, otros pueden requerir formación o acompañamiento adicional.

\subsection{PERCEPCIÓN SOBRE LAS HABILIDADES DE LOS ESTUDIANTES DESPUÉS DE LA INTERVENCIÓN}

Después de la intervención los doctorandos tomaron su tercera materia. La doctora titular del curso ya no tuvo una pareja pedagógica, sino que ella trabajó directamente con la asignación, retroalimentación y evaluación de trabajos escritos. Ella asumió que habían pasado por un proceso formativo previo, por lo que no dedicó tiempo a señalar cómo se debían estructurar los trabajos, solo dio indicaciones de qué esperaba y retroalimentó todas las entregas conforme a lo solicitado.

Según sus impresiones, los estudiantes estaban dispuestos a leer los textos asignados y elaboraban los textos que les solicitaba, siempre con base en las lecturas realizadas. Al construir Montes, Bonilla y Salazar doi: https://doi.org/10.36799/el.v4i1.84 Volumen 4, Número 1, Año 2019, ISSN: $2448-5942$ 
los textos los estudiantes integraban elementos de las lecturas y lo hacían de forma pertinente para integrar otras voces a sus trabajos y para complejizar los temas que exponían. No siempre se presentaban los textos con una estructura y formato perfectos, pero los trabajos evidenciaban que los estudiantes sí tenían nociones sobre cómo estructurar, redactar y presentar los textos.

La docente también sostiene que hay doctorandos que requieren acompañamiento y formación complementaria para que desarrollen las habilidades que les permitan seguir adelante, pero que el grupo en general va por buen camino, está dispuesto y con una actitud favorable para el aprendizaje. Algo que hace falta, en general, es que se apropien del discurso especializado de su área de formación y, sobre todo, que tengan más actividades y experiencias que les permitan practicar lo que han aprendido.

Para complementar la perspectiva de los académicos y de los comentarios generales que se recuperaron de los estudiantes como parte de las acciones de formación, se elaboró un cuestionario y se solicitó a tres estudiantes que lo respondieran. Con respecto a la primera etapa de la intervención, es decir, el curso impartido, los estudiantes señalan que sus aprendizajes principales fueron en torno a los siguientes temas: conocimientos y habilidades para mejorar la redacción, así como lo relacionado con el uso de conectores y la estructura de los textos; algunos detalles del formato APA, particularmente formato de citas y referencias, además de normas para la redacción de textos académicos, el uso adecuado de la gramática para la redacción de ideas claras y precisas, además de que les ayudó a "crear consciencia sobre la escritura de textos académicos”. En cuanto al beneficio de la actividad, los estudiantes señalan que la experiencia "tuvo un significado positivo", pero dos de ellos discrepan en cuanto a la utilidad que los aprendizajes adquiridos han tenido para la elaboración de su tesis. Uno de los estudiantes considera que lo aprendido ha sido benéfico, en sus propias palabras, "ha sido de gran utilidad para mi trabajo de tesis"; además, afirma que el curso le permitió adquirir elementos para hacer correcciones en sus trabajos. Otro estudiante opina que, a pesar de que fue una experiencia de aprendizaje, el momento de la intervención no fue el más apropiado:

Considero valioso lo aprendido, en términos de la preparación de la Dra. $[\mathrm{X}]$; sin embargo, si ubico en el tiempo el significado no es el mismo si en este momento tuviera una orientación de su parte, sobre todo porque ya se estaría trabajando a partir de un documento real de tesis. 
En cuando a la segunda etapa de la intervención, es decir, la pareja pedagógica, los estudiantes consideran que les fue de utilidad para mejorar sus trabajos. Destacan la actividad de revisar trabajos de sus compañeros, pues les sirvió para "mejorar en la comunicación de ideas" y darse cuenta "de las diferentes formas de expresar una idea y apropiarte de ella", además de que identificaron el reto de "que las demás personas entiendan de lo que estás escribiendo". Esta experiencia les posibilitó ver en los demás sus mismos errores, como comentó un estudiante:

Durante clase se dieron algunos ejemplos en donde pude darme cuenta de que cometía los mismos errores y eso me permitió autocorregirme en cuestiones de extensión y estructura de párrafos, así como los elementos de la estructura general de un texto y las ideas claras que se deben desarrollar en cada una de las partes de estas.

A otro estudiante esta experiencia le sirvió para hacerse más consciente de sus producciones escritas: "soy más cuidadosa al momento de estar redactando, leo el documento varias veces en voz alta y tengo mi manual APA a la mano, pero soy estudiante y aun [sic] continúo aprendiendo". El tercer estudiante, en cambio, señala que el problema no es detectar errores, sino identificarlos en los propios trabajos, además de atender las demandas de los directores, que discrepan en lo que entienden por el seguimiento correcto de las normas.

Es relativamente sencillo el identificar los errores, aquí el asunto es que se trabaja y retrabaja sobre un mismo documento y esto hace que se incida en errores de redacción, que solo otro detecta, porque está ajeno al documento.

En cuanto al citado, es todo un tema, porque lees el manual APA, lo aplicas y luego recibes observaciones del tutor y de los directores de tesis, las cuales discrepan entre sí. Por lo tanto, decides hacerle caso al tutor, como todo estudiante a quien van a evaluar.

Finalmente, los estudiantes hacen propuestas sobre cómo mejorar la intervención, para que los aprendizajes adquiridos sean más profundos. Dos de ellos proponen hacer más práctica la primera etapa de la intervención, como se ve en el siguiente fragmento recuperado de las respuestas de un estudiante:

Considero que la práctica hace al maestro, por lo tanto, que se produzca un poco más (documentos pequeños) y que se analicen textos para identificar estilos de escritura en base al tipo de investigación. 
Otro estudiante reitera la necesidad de colocar la intervención en el momento en el que se está trabajando la tesis, tanto para que reciban formación explícita como para que tengan un tutor de escritura, como se ve a continuación:

En conclusión, yo creo que si se ofertara de nuevo el taller de Literacidad quedaría mejor una vez iniciada la tesis o también funcionaría tener sesiones exclusivas en cuanto a dudas de formato, es decir que se pueda contar con un tutor de contenido y otro de formato.

\section{A MANERA DE CIERRE: TAREAS PENDIENTES}

La intervención que se diseñó tuvo dos etapas. La primera, del tipo que Calderón Arévalo y Tapia Ladino (2016) denominaron seminario transversal o emergente, para atender necesidades detectadas en los estudiantes al inicio del programa; la segunda etapa consistió en un programa de pareja pedagógica, con el que un docente de formación disciplinar se hizo acompañar de una tutora de escritura para que orientara y acompañara a los estudiantes en la producción de textos que el curso demandaría. La perspectiva de los estudiantes y de los docentes involucrados es que esta experiencia fue efectiva, a pesar de que comenzó con una intención remedial. Esto se puede afirmar a partir de las recuperaciones hechas por las facilitadoras durante las sesiones de trabajo. No obstante, no debe asumirse como el cierre del proceso, especialmente porque los estudiantes necesitan seguir practicando y seguir recibiendo formación sobre los tipos de textos que el programa les demanda, como quedó de manifiesto en la recuperación que hicieron los estudiantes en el cuestionario. Los doctorandos deben aprender a ser doctores en educación, lo que implica adquirir conocimientos y un vocabulario propio, además de una forma de escribir y de leer, y tener la validación, a través de sus tesis, por parte de especialistas en la temática. Ciertamente muchos de los contenidos abordados en la intervención fueron de tipo normativo o estilístico, y vinculadas con prácticas de literacidad académica, pero se consideró necesario abordarlos en razón de las diferencias en los perfiles y de los conocimientos previos de los estudiantes. Sin duda, habrá que seguir trabajando con la enculturación de los estudiantes al campo en el que están siendo formados.

El paso siguiente será analizar el acompañamiento que los doctorandos reciben por parte de sus directores de tesis, así como recuperar la experiencia de los estudiantes en relación al nivel de adquisición de conciencia comunicativa orientada a su trabajo de tesis doctoral. Esto es de 
particular relevancia por la afirmación de uno de los estudiantes a los que se les aplicó el cuestionario de recuperación, al señalar que los directores no siempre coinciden en relación a las especificaciones de los textos solicitados. Con este seguimiento será posible identificar las prácticas de lectura y escritura en las que están participando, la manera en la que influye el seguimiento que hagan los directores, los retos que enfrentan y la forma en que los resuelven, ya sea teniendo como base lo aprendido con la intervención o recurriendo a medios adicionales.

\section{REFERENCIAS}

Applebee, Arthur N. "Writing and Reasoning". Review of Educational Research, 54 (4), 577-96, 1984. doi: https://doi.org/10.3102/00346543054004577

Ávila Reyes, Natalia, Paula González-Álvarez y Christian Peñaloza Castillo. "Creación de un programa de escritura en una universidad chilena: estrategias para promover un cambio institucional". Revista mexicana de investigación educativa, 18 (57), 537-60, 2013. http://www.comie.org.mx/revista/v2018/rmie/index.php/nrmie/article/view/257/2 $\underline{57}$

Calderón Arévalo, Edith, y Mónica Tapia Ladino. "Sistemas para el apoyo, seguimiento y evaluación de las competencias comunicativas". Revista mexicana de investigación educativa, 21 411-435, 2016. http://www.comie.org.mx/revista/v2018/rmie/index.php/nrmie/article/view/74/74

Camps, Anna. "Prólogo: Comunicar en contextos científicos y académicos. Escribir y comunicarse en contextos científicos y académicos". En Conocimientos y estrategias, editado por Montserrat Castelló, 9-12. España: Graó, 2007.

Carlino, Paula. "Alfabetización académica diez años después". Revista Mexicana de Investigación $\begin{array}{llll}\text { Educativa, } & 18 & \text { (57), } & \end{array}$ http://www.comie.org.mx/revista/v2018/rmie/index.php/nrmie/article/view/250

. Escribir, leer y aprender en la universidad: una introducción a la alfabetización académica. México: Fondo de Cultura Económica, 2006.

. "Exploración de géneros, diario de tesis y revisión entre pares: Análisis de un ciclo de investigación-acción en talleres de tesis de posgrado". En Escritura y producción de Montes, Bonilla y Salaz̧ar doi: https://doi.org/10.36799/el.v4i1.84 Volumen 4, Número 1, Año 2019, ISSN: $2448-5942$ 
conocimiento en las carreras de posgrado, editado por Elvira Narvaja de Arnoux, 227-46. Argentina: Santiago Arcos, 2008.

. "La experiencia de escribir una tesis: contextos que la vuelven más difícil". Anales del Instituto de lingüistica, 24, 1-10, 2005.

. "Leer textos científicos y académicos en la educación superior: obstáculos y bienvenidas a una cultura nueva". Unipluriversidad, 3 (2), 17-23, 2003.

Carrasco Altamirano, Alma, y Rollin L. Kent Serna. "Leer y escribir en el doctorado o el reto de formarse como autor de ciencias”. Revista Mexicana de Investigación Educativa, 16 (51), 1227 1251, 2011.

Carter, Susan, y Vijay Kumar. "Ignoring me is part of learning': Supervisory feedback on doctoral writing". Innovations in Education and Teaching International, 54 (1), 68-75, 2016.

Castro Azuara, María Cristina, y Martín Sánchez Camargo. "Escribir en la universidad: la organización retórica del género tesina en el área de humanidades”. Perfiles Educativos, XXXVI (148), 50-67, 2015.

Fang, Zhihui. "Language correlates of disciplinary literacy". Topics in Language Disorders, 32 (1) 19-34, 2012.

Fernández Fastuca, Lorena, y Catalina Wainerman. "La dirección de tesis de doctorado: ¿una práctica pedagógica?”. Perfiles educativos, 37 (148), 156-71, 2015.

Gee, James Paul. “The new literacy studies. From 'socially situated' to the work of the social. The new literacy studies". En From socially situated to the work of the social, editado por David Barton et al., 180-96. Estados Unidos: Routledge, 2000.

Instituto Tecnológico y de Estudios Superiores de Monterrey. Prueba de Admisión a Estudios de Posgrado (PAEP). 2014, http://sitios.itesm.mx/va/calidadacademica/paep.htm Revisión de la prueba. s. f., http://sitios.itesm.mx/va/calidadacademica/files/revision.pdf

Miras, Mariana. "La escritura reflexiva. Aprender a escribir y aprender acerca de lo que se escribe”. Infancia y Aprendizaje. Journal for the Study of Education and Development, 23 (89), 6580, 2014. 
Miras, Mariana, e Isabel Solé. "La elaboración del conocimiento científico y académico. Escribir y comunicarse en contextos científicos y académicos". En Conocimientos y estrategias, editado por Montserrat Castelló, 83-112. España: Graó, 2007.

Moje, Elizabeth Birr. "Developing socially just subject-matter instruction: a review of the literature on disciplinary literacy teaching". Review of research in education, 31 (1), 1-44, 2007. doi: https://doi.org/10.3102/0091732X07300046001

Olson, David R. The World on Paper: The Conceptual and Cognitive Implications of Writing and Reading. Reino Unido: Cambridge University Press, 1996.

Ortega Ruiz, Pedro, y Alberto Gárate Rivera. Una escuela con rostro humano. CETYS Universidad, 2017.

Padilla, Constanza. "Desafíos epistémicos y argumentativos en la escritura de postgrado: Géneros científico-académicos y trayectorias de maestrandos y doctorandos". Traslaciones, 3 (6), 165-96, 2016.

Serrano, Stella. "La lectura, la escritura y el pensamiento. Función epistémica e implicaciones pedagógicas”. Lenguaje, 42 (1), 97-122, 2014.

Shanahan, Timothy, y Cynthia Shanahan. "Teaching disciplinary literacy to adolescents: Rethinking content-area literacy". Harvard Educational Review, 78 (1), 40-59, 2008. doi: https://doi.org/10.17763/haer.78.1.v62444321p602101

Street, Brian V. "New literacy studies". En Language, ethnography, and education: bridging new literacy studies and Bourdieu, editado por Michael Grenfell et al., 27-49. Estados Unidos: Routledge, 2013.

Zavala, Virginia. 'La literacidad, o lo que la gente 'hace' con la lectura y la escritura". Textos de Didáctica de la Lengua y la Literatura, 47, 71-79, 2008. 